\title{
An intervention to increase earthquake and fire preparedness
}

\author{
G. Perez-Fuentes \& H. Joffe \\ Department of Psychology and Language Sciences, \\ University College London, UK
}

\begin{abstract}
Levels of natural hazard preparedness continue to be low across cultures. Studies on natural hazard preparedness have consistently found that simply providing people with information about risk is not sufficient to change preparedness behaviours. Research in the field of social representations and emergency preparedness indicate that it is a combination of cognitive, emotional, and cultural factors that affect preparedness behaviours. Therefore, understanding how personal, social, and cultural dynamics influence people's interpretations of risk is essential if one is to intervene effectively in hazard preparedness. The existing natural hazard preparedness literature contains two major shortcomings. Firstly, studies of community emergency preparedness interventions are scarce. Secondly, the majority of these studies are imprecisely described; many lack detailed information regarding the study's procedures and the content of the interventions. Such work hinders development of the field of natural hazard preparedness: replication of interventions is difficult and publics are subjected to interventions with little empirical support. In order to develop the field of hazard preparedness, a multidisciplinary team of researchers aims to design, conduct and evaluate a rigorous cross-cultural intervention for fire and earthquake preparedness. The present study will explore the different cognitive, emotional, and cultural factors that play a role in emergency preparedness with the goal of improving earthquake and fire emergency preparedness behaviours among lay people.
\end{abstract}

Keywords: preparedness, natural hazards, intervention, earthquake, fire, community resilience, behaviour change. 


\section{Introduction}

It is critical to adopt and maintain preparedness measures at the household level if risk of injury and damage at home is to be reduced. Such measures also minimise the disruption that follows a natural disaster. Disaster preparedness measures range from securing heavy objects, structural retrofitting, and storing food and water, to having communication and evacuation plans. It is known that a prepared community recovers faster and more effective after a disaster (Mileti et al. [1]). This, in turn, translates into a more resilient public that is able to effectively respond before, during, and after the disaster (Lindell et al. [2]). Preparedness or readiness thus constitutes the first phase of resilience. In a world where globalization, gentrification, and climate change are rapidly increasing, the building of resilience is critical.

While it is in people's best interests to make safety-related plans before a disaster occurs, the existing literature shows that most people are not prepared for action in emergency situations [3-9]. Even people who live in areas where natural disasters occur frequently are not prepared [10-14]. Authorities have frequently attributed the lack of preparedness among communities to a lack of information. Thus, according to this model, sometimes termed the deficit model [15-19], it is often believed that the provision of hazards information to the public encourages preparation. Nonetheless, studies have consistently found that merely providing people with information about risks and their consequences is not sufficient to affect preparedness behaviours [4-6, 20-22]. Furthermore, previous experience with natural disasters has not been found to be a good predictor of preparedness [12, 23-29]. Hence, simply being aware of the risk, in particular earthquake risk does not increase the propensity to undertake protective behaviours (Solberg et al. [30]).

In the past years, research has attempted to understand what influences and predicts people's preparedness behaviours [5, 6, 21, 31]. A few studies have focused their efforts on interventions to improve preparedness behaviours at the household level, with little success. In addition, their procedures and methods are imprecisely described and evaluated. This paper summarizes a review of current community interventions on earthquake and fire preparedness, and then describes our Challenging Risk project, a multidisciplinary, cross-cultural, community intervention for earthquake and fire preparedness.

\section{The social psychological literature on natural hazard preparedness}

The psychology of risk field has increasingly accepted that perceptions are influenced by emotional and sociocultural factors [32], rather than purely rational factors; such perceptions then drive behaviour [10]. Along with the host of cognitive biases that colour risk perception stands a wide range of emotional and sociocultural factors (e.g., anxiety, trust and fatalism) that mediate the execution of preparedness actions. 


\subsection{Predictors of preparedness}

Cognitive biases, such as optimistic bias, where people believe that they are less at risk of being affected by a danger when compared to others, or normalization bias, which postulates that people who experience little or no harm in an earthquake are less likely to heed future earthquake warnings, affect how risk is perceived by the public and hinder preparedness [10, 24, 33]. By way of contrast the following have been reported to be good predictors of preparedness: behavioural intention [20,34], perceived self-efficacy [31], collective efficacy [31], empowerment [31], perceived outcome expectancy [31], critical awareness $[21,31]$, social cohesion $[21,35,36]$, sense of community $[9,37]$, community participation $[21,31,35,36,38,39]$, and trust in the authorities [31, 40]. Nevertheless, the relationship between awareness and preparedness is complex and there are several variables that have been reported to affect this relationship. Emotional and sociocultural variables, such as such as anxiety, trust and fatalism, moderate the relationship between hazard awareness and actual preparedness behaviours [10, 34, 41-43], as well as personal responsibility [4-6, 20]. Research has also shown that being a home owner versus renter and having children or dependents increases seismic adjustment $[5,44]$.

In summary, it seems that people's interpretation of their risk, their feeling about it, their sense of their own efficacy, and that of their community are far more central than awareness of it, in determining their preparedness actions. Consequently, studying how personal, social, and cultural factors influence how people interpret risk is essential if we are to intervene in hazard preparedness.

\section{Intervention studies on earthquake and fire preparedness}

The literature on earthquake and fire community preparedness interventions is scarce. Despite there being a wide range of mass media and internet-based natural disaster preparedness sites, documentation and evaluation of them is rare. An additional problem is that, when documented and/or evaluated, most are vaguely described. Furthermore, few have been proven to demonstrate increased disaster preparedness behaviours at the household level. Therefore, more explicit and better designed natural hazard preparedness interventions are needed so that they can be replicated and improved. The goal would be to engage publics in interventions based within strong empirical evidence.

An online Google Scholar search with the words "natural hazards intervention", "natural disaster preparedness" and "preparedness intervention" of earthquake community preparedness intervention studies yielded a result of nine studies. Four of them were on earthquakes and other natural hazards, such as landslides and/or floods [11, 45-47], two focused solely on earthquakes [48, 49], one on cyclones [50], and two on disasters in general [51,52]. Studies were conducted in Turkey [11], Martinique [48], Los Angeles, USA [51, 52], Australia [50], Iran [45], Pakistan [49], New Zealand [46] and Taiwan [47]. Some of the studies were conducted during critical time periods [11, 47, 50]. Some studies targeted vulnerable populations, such as children, teachers and 
parents and were conducted in schools [46, 49] and two studies were done on low-income minorities $[51,52]$. The studies contained a number of limitations. Some studies did not describe the actual content of their intervention [11, 47, 49, 50]. Two of them did not evaluate the intervention's [47, 49] effectiveness. Furthermore, some studies did not use control groups [46, 51, 52], leading to unreliable results. Overall, most of the methods, including materials, recruitment of participants and measures used, are not clearly described. Regarding their generalizability, several used respondent driven sampling leading to homogeneous samples, and therefore, sample bias [51, 52]. Finally, regarding a theoretical orientation, most were not explicit about containing one, though others used a theoretical model for their interventions [11, 46, 50, 51]. Leaving aside these limitations, some of the ingredients of successful interventions can be found among these studies. Overall, earthquake preparedness interventions proved successful in affecting adjustment measures when including hands-on training, face-to-face interactions, and those that targeted empowerment and community cohesion.

The literature on home fire preparedness is larger than the one on earthquakes, with most of the studies conducted in the U.S. [53-58]. A review of home fire preparedness interventions studies showed that most of them focused on smoke alarm canvassing and smoke alarm installations, which proved to be among the most effective interventions to improve fire preparedness behaviours as well as reducing fire related deaths and injuries [54, 57]. In addition, the presence of fire service personnel appears to be the most effective method of distributing smoke alarms Douglas et al. [59]. Again hands-on training was the most effective in improving preparedness responses Miller et al. [58]. Nonetheless, studies have consistently shown that the level of preparation for fire hazards tends to be poor $[56,58,60]$. This is consistent with existing literature on fire preparedness in the U.S. [58, 61, 63]. For instance, having a smoke alarm was found to reduce the risk of death by $40 \%-50 \%$. However, $40 \%$ of fires reported to fire departments occur in homes without alarms and $70 \%$ of fire deaths occur in homes with either no smoke alarm or where the alarm malfunctioned (Ballesteros and Kresnow [60]). The functionality of alarms remains a problem $[54,56]$, and so does the lack of rigor in the evaluation designs of these studies.

Most of these interventions on fire or on earthquake preparedness, when evaluated, were based on self-report measures only, such as surveys and questionnaires. In contrast, our intervention aims to include home visits and review images and documentation of their preparedness behaviours, in addition to administering self-report measures.

\section{Designing an intervention on earthquake and fire preparedness}

The existing approach to risk continues to be too specific to particular hazards. Authorities take into account the possibility of earthquakes, tsunamis, fires, and the collapse of systems, but they view and handle them separately. To our 
knowledge there is no published community intervention study that combines earthquake and home fire safety preparedness measures. By including both hazards together in one intervention, we develop an integrated multihazard preparedness approach [63]. In addition, by including home fire preparedness, a hazard with higher incidence than earthquakes and, therefore, one that the public has experienced and witnessed, the more everyday risk gets paired with the longer return period risk. This may facilitate a more everyday routine of adopting disaster preparedness measures at home.

According to Michie et al. [69] behaviour change interventions need to be based on theoretical models of behaviour that explain the behaviour change process. The field of natural hazard preparedness has used several theoretical models in their interventions, such as the person-relative-to-event-model (Duval and Mulilis [5]), the precaution adoption process model (Weinstein and Sandman [64]), and the emotion focused coping model (Lazarus and Folkman [65]). Models with proven success in the prediction of preparedness behaviours are the theory of planned behaviour and protection motivation theory [66, 67], and they provide a strong basis for developing an intervention on natural hazard preparedness. Recent studies have attempted to develop models to predict the adoption of natural hazard preparedness with good success [21, 68]. Existing models of natural hazard preparedness largely rely on more rational factors tapped by the theory of planned behaviour and protection motivation theory, and this has been proven to be not enough to explain preparedness behaviours. Thus including just cognitive factors is not enough to understand this relationship between risk awareness and hazard preparedness.

In a separate line of enquiry, it has been found that understanding the sociocultural factors that affect preparedness behaviours in a community is essential to intervene in them. Joffe et al. [10] interviewed a sample of lay people in Seattle, Washington; Osaka, Japan; and Izmir, Turkey, and found that, consistent with existing literature, awareness of seismic adjustment behaviour was not translated into action. They found that the majority of respondents in Seattle felt less at risk for earthquakes than their counterparts in the area of San Andreas Fault due to a perceived geographical distance from the threat. Osaka respondents also felt they were less at risk for earthquakes, compared to people living in developing countries, arguing for Japan's advanced technology. In Izmir, people felt largely defeatist regarding preparedness largely because of their extreme lack of trust in their government and builders, regarding the solidity of their buildings, as well as the potential to get assistance and aid in the event of an earthquake. In addition, those cultures with higher levels of anxiety in relation to earthquakes prepared less, while those with lower anxiety and even a positive sense of awe in relation to earthquakes engaged in more adjustment measures. Furthermore, those with least trust in their societal institutions prepared least with those with more trust preparing more. Finally, individuals with higher fatalism tended to prepared less in contrast to those with an 'I can' attitude, who tended to prepared more.

Grounded in this work on social representations of earthquakes, and having reviewed the existing literature on earthquake and fire preparedness and on 
behaviour change interventions, this study aims to conduct a cross-cultural intervention on earthquake and fire preparedness behaviours in Seattle, United States and Izmir, Turkey. It will analyse the effect of the interventions in the targeted communities to see if these can bring about behaviour change. We will use a modified version of Paton's model of natural hazard risk reduction preparedness [21, 68], which proposes motivation, outcome expectancy, and self-efficacy beliefs as main predictors of preparedness. Paton adds a fourth variable to his model, intentions to prepare; however, since the literature on intentions as a predictor of behaviour seems unclear, we will leave this fourth variable out. Instead, we will incorporate the emotive variables of anxiety and trust, as well as sociocultural variables, such as sense of responsibility, empowerment and social cohesion into the intervention as predictors of preparedness. The proposed intervention will target the following determinants of behaviour, self-efficacy, outcome expectancy, and motivation, through different behavioural techniques. First, in order to increase motivation we will use rewards, incentives, graded tasks, social encouragement and support, as well as persuasive communication as behaviour change techniques (Michie et al. [69]). In order to affect perceived self-efficacy, techniques proven to be effective in this behavioural domain, such as self-monitoring, rehearsal, coping skills, graded tasks, social encouragement and support, and feedback will be employed. Lastly, to increase perceived outcome efficacy, we will employ persuasive communication and feedback, as proven effective techniques of behaviour change.

\subsection{Methods}

The proposed intervention will be conducted first in Seattle, U.S.A, in the summer of 2015, and then in Izmir, Turkey, following up the previous study done by Joffe in these two coastal cities with high seismic risk. Study objectives are to increase household preparedness measures for earthquakes and home fires in lay people and to evaluate changes in their levels of motivation, self-efficacy, perceived outcome, trust, empowerment, anxiety, and social cohesion, as well as levels of adjustment measures, before and after the intervention. The study will use a non-randomised control, longitudinal intervention, with pre-test and posttest design. In order to assess the effects of the intervention we will have a control group, which will consist of people from a neighbourhood geographically separated from the intervention one. Individuals in both groups will fill out an online questionnaire to assess their baseline level of preparedness. Following the completion of the questionnaire, participants in the intervention group will complete a workshop on fire and earthquake preparedness. Directly after the intervention as well as three and 12 months one, both groups will fill out the questionnaire to assess intervention effects. The intervention group will participate in a six-hour interactive, face-to face, hands-on practice workshop, divided in two afternoons, and led by an expert in emergency management training. The workshop will have approximately 30 people each and it will include hands-on training, as well as using interactive tools, such as uploading their photos and videos on social media sites, to demonstrate adjustment 
behaviours. In addition, participants will receive home visits from the fire department and one of our researchers, who will ask them to demonstrate some of their adjustment behaviours in their household (e.g., test the smoke alarms, show how they secured heavy objects).

\subsubsection{Sample}

The sample for the study in both Seattle and Izmir will be 200 adults recruited using professional recruitment companies to enlist matched samples of 100 participants from each neighbourhood, who will then be assigned to each group, intervention or control. Neighbourhood selection has been carefully done by a team of multidisciplinary researchers (structural engineers, experts in citizen science, and psychologists) who cautiously reviewed census data and other governmental and local data and travelled to Seattle for 15 days to visit different neighbourhoods and ultimately select two that are sociodemographically representative of the city. The same will be done for Izmir.

\subsubsection{Measures}

Empowerment, perceived self-efficacy and perceived group efficacy, perceived outcome expectancy, anxiety, trust, fatalism, and demographics will be assessed in the questionnaire. Preparedness behaviours will be assessed by the questionnaire, with 19 items measuring earthquake adjustments and 16 on fire safety. In addition, in between workshops, an expert from the fire department and one of the co-leaders of the workshops will make home visits to the houses that participated in the workshop, to evaluate preparedness measures and assist individuals with their implementation if they are having questions or problems.

\section{Conclusions}

The field of natural hazard disaster preparedness is in need of better designed interventions on natural hazard preparedness in order to engage the public in successful interventions. In addition, recent studies emphasize the need for a multihazard approach to emergency preparedness interventions. A public that is better prepared for multiple hazards is better prepared for specific and unpredictable hazards, and is therefore more resilient. To our knowledge this is the first intervention that combines earthquake and home fire preparedness, and that aims to compare results among different cultures, Seattle, USA and Izmir, Turkey. In addition, the this intervention was carefully designed by a team of multidisciplinary researchers, from the fields of structural engineering, citizen science and social psychology, who previously evaluated the social representations of earthquakes in lay people in Seattle and Izmir (Joffe et al. [10]). Results of these thorough interviews have allowed this team to develop detailed interventions tailored to match the social representations of each location. This study has significant implications for the field of natural disaster preparedness at an international level as well as for the area of interventions on natural hazard preparedness, as it will allow for replication, improvement, and therefore development of the field. 


\section{References}

[1] Mileti, D.S., Drabek, T.E. \& Haas, J.E., Human systems in extreme environments: A sociological perspective, vol. 21: Institute of Behavioral Science, University of Colorado, 1975.

[2] Lindell, M.K., Tierney, K.J. \& Perry, R.W., Facing the Unexpected: Disaster Preparedness and Response in the United States: Joseph Henry Press, 2001.

[3] Lambert, A.J., Burroughs, T. \& Nguyen, T., Perceptions of risk and the buffering hypothesis: The role of just world beliefs and right-wing authoritarianism, Personality and Social Psychology Bulletin, 25, pp. 643$65,1999$.

[4] Ballantyne, M., Paton, D., Johnston, D., Kozuch, M. \& Daly, M., Information on volcanic and earthquake hazards: The impact on awareness and preparation, Institute of Geological and Nuclear Sciences Limited Science Report, Wellington, 2000.

[5] Duval, T.S. \& Mulilis, J.P. A Person-Relative-to-Event (PrE) approach to negative threat appeals and earthquake preparedness: A field study, Journal of Applied Social Psychology, 29, pp. 495-516, 1999.

[6] Lindell, M.K. \& Whitney, D.J., Correlates of household seismic hazard adjustment adoption, Risk Analysis, 20, pp. 13-26, 2000.

[7] McClure, J., Walkey, F. \& Allen, M., When earthquake damage is seen as preventable: Attributions, locus of control and attitudes to risk, Applied Psychology, 48, pp. 239-256, 1999.

[8] Mulilis, J.P. \& Duval, T.S., Negative threat appeals and earthquake preparedness: A Person-Relative-to-Event (PrE) model of coping with threat, Journal of Applied Social Psychology, 25, pp. 1319-1339, 1995.

[9] Paton, D., Emergency planning: Integrating community development, community resilience and hazard mitigation, Journal of the American Society of Professional Emergency Managers, 7, pp. 109-118, 2000.

[10] Joffe, H., Rossetto, T., Solberg, C. \& O'Connor, C., Social representations of earthquakes: A study of people living in three highly seismic areas, Earthquake Spectra, 29, pp. 367-397, 2013.

[11] Karanci, A.N., Aksit, B. \& Dirik, G., Impact of a community disaster awareness training program in Turkey: Does it influence hazard-related cognitions and preparedness behaviors, Social Behavior and Personality: an international journal, 33, pp. 243-258, 2005.

[12] Rüstemli, A. \&. Karanci, A.N., Correlates of earthquake cognitions and preparedness behavior in a victimized population, The Journal of Social Psychology, 139, pp. 91-101, 1999.

[13] Faupel, C.E., Kelley, S.P. \& Petee, T., The impact of disaster education on household preparedness for Hurricane Hugo, International Journal of Mass Emergencies and Disasters, vol. 10, pp. 5-24, 1992.

[14] Garcia, E.M., Earthquake preparedness in California: A survey of Irvine residents: National Emergency Training Center, 1989. 
[15] Smith, K., Environmental hazards: assessing risk and reducing disaster: Routledge, 2013.

[16] Bauer, M., Durant, J. \& Evans, G., European public perceptions of science, International Journal of Public Opinion Research, 6, pp. 163-186, 1994.

[17] Evans, G. \& Durant, J., The relationship between knowledge and attitudes in the public understanding of science in Britain, Public Understanding of Science, 4, pp. 57-74, 1995.

[18] Eden, S., Public participation in environmental policy: considering scientific, counter-scientific and non-scientific contributions, Public understanding of science, 5, pp. 183-204, 1996.

[19] Sturgis, P.J. \& Allum, N., Gender differences in scientific knowledge and attitudes toward science: reply to Hayes and Tariq, Public Understanding of Science, 10, pp. 427-430, 2001.

[20] Paton, D., Smith, L.M. \& Johnston, D., Volcanic hazards: Risk perception and preparedness, New Zealand Journal of Psychology, 29, pp. 86-91, 2000.

[21] Paton, D., Smith, L. \& Johnston, D., When good intentions turn bad: Promoting natural hazard preparedness, 2005.

[22] Perry, R.W. \& Lindell, M.K., Volcanic risk perception and adjustment in a multi-hazard environment, Journal of Volcanology and Geothermal Research, 172, pp. 170-178, 2008.

[23] Plapp, T. \& Werner, U., Understanding risk perception from natural hazards: examples from Germany, Risk, 21, pp. 101-108, 2006.

[24] Johnston, D.M., Bebbington Chin-Diew Lai, M.S, Houghton, B.F. \& Paton, D., Volcanic hazard perceptions: comparative shifts in knowledge and risk, Disaster Prevention and Management: An International Journal, 8, pp. 118-126, 1999.

[25] Rincon, E., Linares, M.Y. \& Greenberg, B., Effect of previous experience of a hurricane on preparedness for future hurricanes, The American Journal of Emergency Medicine, 19, pp. 276-279, 2001.

[26] Lindell, M.K. \& Prater, C.S., Household adoption of seismic hazard adjustments: A comparison of residents in two states, International Journal of Mass Emergencies and Disasters, 18, pp. 317-338, 2000.

[27] Karanci, N.A. \& Aksit, B., Strengthening community participation in disaster management by strengthening governmental and nongovernmental organisations and networks: A case study from Dinar and Bursa (Turkey), 1999.

[28] Palm, R., Urban earthquake hazards: the impacts of culture on perceived risk and response in the USA and Japan, Applied Geography, 18, pp. 3546, 1998.

[29] Dooley, D., Catalano, S., Mishra, S. \& Serxner, S., Earthquake preparedness: Predictors in a community survey1, Journal of Applied Social Psychology, 22, pp. 451-470, 1992. 
[30] Solberg, C., Rossetto, T. \& Joffe, H., The social psychology of seismic hazard adjustment: re-evaluating the international literature, Natural Hazards and Earth System Sciences, 10, pp. 1663-1677, 2010.

[31] Paton, D. \& Johnston, D., The Christchurch earthquake: Integrating perspectives from diverse disciplines, International Journal of Disaster Risk Reduction, 2015.

[32] Slovic, P., The feeling of risk: New perspectives on risk perception: Routledge, 2010.

[33] Mileti, D.S. \&. O’Brien, P.W, Warnings during disaster: Normalizing communicated risk, Social Problems, 39, pp. 40-57, 1992.

[34] Paton, D., Bajek, R., Okada, N. \& McIvor, D., Predicting community earthquake preparedness: a cross-cultural comparison of Japan and New Zealand, Natural Hazards, 54, pp. 765-781, 2010.

[35] McGee, T.K. \& Russell, S., "It's just a natural way of life..." an investigation of wildfire preparedness in rural Australia, Global Environmental Change Part B: Environmental Hazards, 5, pp. 1-12, 2003.

[36] Tierney, K.J., Lindell, M.K. \& Perry, R.W., Facing the Unexpected: Disaster Preparedness and Response in the United States. Washington, DC.: Joseph Henry Press, 2001.

[37] Bishop, B., Paton, D., Syme, G. \& Nancarrow, B., Coping with environmental degradation: Salination as a community stressor, Network, 12, pp. 1-15, 2000.

[38] Karanci, N.A. \& Aksit, B., Building disaster-resistant communities: Lessons learned from past earthquakes in Turkey and suggestions for the future, International Journal of Mass Emergencies and Disasters, 18, pp. 403-416, 2000.

[39] Perry, R.W. \& Lindell, M.K., Preparedness for emergency response: guidelines for the emergency planning process, Disasters, 27, pp. 336-350, 2003.

[40] Dillon, J. \& Phillips, M., Social capital discussion paper, Unpublished manuscript, Curtin University, Perth, Western Australia, 2001.

[41] Johnston, D., Becker, J., McClure, J., Paton, D., McBride, S., Wright, K. et al., Community Understanding of, and Preparedness for, Earthquake and Tsunami Risk in Wellington, New Zealand, in Cities at Risk. vol. 33, H. Joffe, T. Rossetto, and J. Adams, Eds., Springer Netherlands, 2013, pp. 131-148.

[42] Paton, D., Risk communication and natural hazard mitigation: how trust influences its effectiveness, International Journal of Global Environmental Issues, 8, pp. 2-16, 2008.

[43] Johnston, D.M., Tabulated results of the 2003 national coastal community survey vol. 2003: Institute of Geological \& Nuclear Science, 2003.

[44] Turner, R.H., Nigg, J.M. \& Paz, D.H., Waiting for disaster: Earthquake watch in California: Univ of California Press, 1986.

[45] Ardalan, A., Mowafi, H., Malekafzali Ardakani, H., Abolhasanai, F., Zanganeh, A.M., Safizadeh, H. et al., Effectiveness of a primary health care program on urban and rural community disaster preparedness, Islamic 
Republic of Iran: A community intervention trial, Disaster medicine and public health preparedness, 7, pp. 481-490, 2013.

[46] Ronan, K.R. \&. Johnston, D.M, Hazards education for youth: A quasiexperimental investigation, Risk analysis, 23, pp. 1009-1020, 2003.

[47] Chen, L.-C., Liu, Y.-C. \& Chan, K.-C., Integrated community-based disaster management program in Taiwan: a case study of Shang-An village, Natural Hazards, 37, pp. 209-223, 2006.

[48] Audru, J.-C., Vernier, J.-L., Capdeville, B., Salindre, J.-J. \& Mouly, É., Preparedness actions towards seismic risk mitigation for the general public in Martinique, French Lesser Antilles: a mid-term appraisal, Natural Hazards and Earth System Science, 13, pp. 2031-2039, 2013.

[49] New World Hope Organization, Earthquake safety and preparedness program, http://www.newworldhope.org/School\%20Safety.html

[50] Morrissey, S.A. \& Reser, J.P., Evaluating the effectiveness of psychological preparedness advice in community cyclone preparedness materials, 2003.

[51] Glik, D.C., Eisenman, D.P., Zhou, Q., Tseng, C.-H. \& Asch, S.M., Using the Precaution Adoption Process model to describe a disaster preparedness intervention among low-income Latinos, Health education research, 29, pp. 272-283, 2014.

[52] Eisenman, D.P., Glik, D., Gonzalez, L., Maranon, R., Zhou, Q., Tseng, C.H. et al., Improving Latino disaster preparedness using social networks, American journal of preventive medicine, 37, pp. 512-517, 2009.

[53] McDonald, E., Gielen, A., Sleet, D. \& DiClement, R., House fires and other unintentional home injuries, Injury and violence prevention: behavioral science theories, methods, and applications, pp. 274-296, 2006.

[54] Ta, V.M., Frattaroli, S., Bergen, G. \& Gielen, A.C., Evaluated community fire safety interventions in the United States: a review of current literature, Journal of community health, 31, pp. 176-197, 2006.

[55] Thompson, C.J., Jones, A.R., Davis, M.K. \& Caplan, L.S., Do smoke alarms still function a year after installation? A follow-up of the GetAlarmed Campaign, Journal of community health, 29, pp. 171-181, 2004.

[56] DiGuiseppi, C., Roberts, I., Wade, A., Sculpher, M., Edwards, P., Godward, C. et al., Incidence of fires and related injuries after giving out free smoke alarms: cluster randomised controlled trial, Bmj, 325, p. 995, 2002.

[57] Mallonee, S., Istre, G.R., Rosenberg, M., Reddish-Douglas, M., Jordan, F., Silverstein, P. et al., Surveillance and prevention of residential-fire injuries, New England journal of medicine, 335, pp. 27-31, 1996.

[58] Miller, T.R., Bergen, G., Ballesteros, M.F., Bhattacharya, S., Gielen, A.C. \& Sheppard, M.S., Increasing smoke alarm operability through theorybased health education: a randomised trial, Journal of epidemiology and community health, 68, pp. 1168-1174, 2014.

[59] Douglas, M.R., Mallonee, S. \& Istre, G.R., Comparison of community based smoke detector distribution methods in an urban community, Injury Prevention, 4, pp. 28-32, 1998. 
[60] Ballesteros, M.F. \& Kresnow, M.-J., Prevalence of residential smoke alarms and fire escape plans in the US: results from the Second Injury Control and Risk Survey (ICARIS-2), Public health reports, 122, p. 224, 2007.

[61] Istre, G.R., McCoy, M.A., Moore, B.J., Roper, C., Stephens-Stidham, S., Barnard, J.J. et al., Preventing deaths and injuries from house fires: an outcome evaluation of a community-based smoke alarm installation programme, Injury prevention, 20, pp. 97-102, 2014.

[62] Poehlman, J., Flanigan, T., Brown, D., Arnold, S. \& Cutbush, S., Evaluation of the effectiveness of the Smoke Alarm Installation and Fire Safety Education (SAIFE) Program: final report, Research Triangle Park, NC: RTI International, 2010.

[63] Scawthorn, C., Blais, N., Seligson, H., Tate, E., Mifflin, E., Thomas W. et al., HAZUS-MH flood loss estimation methodology. I: Overview and flood hazard characterization, Natural Hazards Review, 7, pp. 60-71, 2006.

[64] Weinstein, N.D. \& Sandman, P.M., A model of the precaution adoption process: evidence from home radon testing, Health psychology, 11, p. 170, 1992.

[65] Lazarus, R.S. \& Folkman, S., Stress, Appraisal, and Coping, New York: Springer 1984.

[66] Abraham, C., Sheeran, P. \& Johnston, M., From health beliefs to selfregulation: Theoretical advances in the psychology of action control," Psychology and Health, 13, pp. 569-591, 1998.

[67] Bennett, P. \& Murphy, S., Psychology and health promotion. Buckingham: Open University Press, 1997.

[68] Paton, D. \& N.Z.E. Commission, Developing a Model to Predict the Adoption of Natural Hazard Risk Reduction and Preparatory Adjustments: Earthquake Commission, 2001.

[69] Michie, S., Johnston, M., Francis, J., Hardeman, W. \& Eccles, M., From theory to intervention: mapping theoretically derived behavioural determinants to behaviour change techniques, Applied psychology, 57, pp. 660-680, 2008. 